\title{
Differenzen zeigender Ethnographie
}

\section{Blickschneisen und Schnittstellen der Kamera-Ethnographie}

\section{Von Bina Elisabeth Mohn}

Zusammenfassung: Wie gelingt eine zeigende Ethnographie? Welche methodologischen Implikationen hat ein an Prozessen des Sehens und Zeigens orientiertes ethnographisches Forschen? Dies skizziert der Text aus der Perspektive der Kamera-Ethnographie (Mohn). Von einer Kamera wird erwartet, dass sie technische Reproduktionen liefert, die dem Abgebildeten mehr oder weniger gleichen. Im Rahmen einer kamera-ethnographischen Methodologie erweist sich jedoch gerade die Entfaltung beobachtender Differenz als Qualitätskriterium ethnographischer Verbildlichungsprozesse. Die differenztheoretisch verstandene Entwicklung von Blickschneisen ermöglicht Selektionen beim Kamera-Gebrauch und unterscheidet sich darin grundlegend von einer mit ,dokumentierender" Kamera arbeitenden sozialwissenschaftlichen Empirie. Der Text fächert das Postulat der Differenz auf: Differenzen des Visuellen, der Blicke und Bilder, des Gegenstandes und der Situation werden angesprochen.

\section{Einleitung}

„Die verschiedenen Methoden, die Ethnographen verwenden, fokussieren nicht ein Phänomen aus unterschiedlichen Blickwinkeln und liefern damit ein (relativ) vollständiges Bild des Geschehens, sondern bringen unterschiedliche Phänomene hervor; das Phänomen ergibt sich folglich durch die Methoden " (Kalthoff 2006: 155).

Wir können davon ausgehen, dass das Vorhaben eines „dichten Zeigens“ in diesem Sinne neue Phänomenbereiche eröffnet. In meinem Beitrag skizziere ich aus der Perspektive der KameraEthnographie (Mohn $)^{1}$ methodologische Implikationen einer an Prozessen des Sehens und Zeigens orientierten ethnographischen Forschung. Was macht die ethnographische Qualität visuellen Forschens aus? Wie gelingt ethnographisches Hinschauen und Herzeigen? Von einer Kamera wird erwartet, dass sie technische Reproduktionen liefert, die dem Abgebildeten mehr oder weniger gleichen und auch ethnographisches Beschreiben wird häufig im Rahmen eines Gleichheitsparadigmas betrieben: Einfach registrieren und notieren, „was ist“. Im Rahmen einer kamera-ethnographischen Methodologie erweist sich hingegen gerade die Entfaltung beobachtender Differenz als Qualitätskriterium. ${ }^{2}$ Die differenztheoretisch verstandene Entwicklung von Blickschneisen ermöglicht begründete Selektionen beim Kamera-Gebrauch und unterscheidet sich darin grundlegend von einer mit „dokumentierender“ Kamera arbeitenden sozialwissenschaftlichen Empirie, die vorrangig den abbildungstechnischen Aspekt von Auf-

1 Neben dem Buch „Filming Culture. Spielarten des Dokumentierens nach der Repräsentationskrise“ (Mohn 2002) liegen mehrere einführende Texte zur Kamera-Ethnographie vor, die unterschiedlich akzentuiert sind: Forschung mit der Kamera (Mohn / Amann 1998); Vom Blickentwurf zur Denkbewegung (Mohn 2007); Die Kunst des dichten Zeigens (Mohn 2008 a); Im Denkstilvergleich entstanden (Mohn 2008 b); Zwischen Blicken und Worten (Mohn 2010); Methodologie des forschenden Blicks (Mohn 2011).

2 Amann / Mohn haben zum Thema „Differenzen zeigender Ethnographie: Blickschneisen und Schnittstellen der Kamera-Ethnographie“ eine Forschungswerkstatt angeboten (19.11.2011, Tagung „Ethnographie und Differenz in pädagogischen Feldern“, Institut für Pädagogik, Friedrich-Alexander-Universität Erlangen-Nürnberg / DGfE-Kommission „Qualitative Bildungs- u. Biographie Forschung“). Mein Beitrag knüpft an die gemeinsame Präsentation an. Klaus Amann danke ich für kritisches Gegenlesen und hilfreiche Anregungen zum vorliegenden Text und seine kontinuierliche Mitwirkung am Erproben und Konzipieren kamera-ethnographischer Methodologie.

Soziale Welt 64 (2013), S. $171-189$ 
zeichnung und Wiedergabe zu nutzen weiß. Sich beim Forschen von Beginn an zwischen dem Blicken, Zeigen, Reden und Schreiben hin und her zu bewegen und dabei tief in das Feld des noch nicht, gar nicht oder nicht mehr Sprachlichen zu geraten, hierin sehe ich ein besonderes Potential der Kamera-Ethnographie. Sie ermöglicht folgenreiche Akzentverschiebungen im ethnographischen Mediengebrauch: vom Abfilmen zum Verbildlichen, vom Audiomitschnitt zum Erlauschen und Vertonen, vom Niederschreiben zum in Worte fassen. Erkundung und Entwurf treten anstelle von Datenerhebung und Rekonstruktion. Im Folgenden wird das Postulat der Differenz zeigender Ethnographie aufgefächert und Differenzen des Visuellen, der Blicke und Bilder, des Gegenstandes und der Situation angesprochen.

\section{Differenz des Visuellen}

Inmitten des Visuellen herrscht Bildlosigkeit. Ethnographisches Beschreiben und Zeigen bedarf einer Methode des Hinschauens, um Notizen und Texte, Bilder und Bildfolgen, Töne und Hörstücke so zu entwerfen, dass sie zur Erkundung und Analyse von Forschungsfeldern taugen. Ein kamera-ethnographisches Forschen ist dabei nicht weniger beobachterabhängig als der ethnographische Schreibprozess. Anstelle der Instrumente Notizbuch und Stift und einer Praxis des Schreibens von Texten im Medium der Schrift werden andere Instrumente und Forschungspraktiken gewählt, Kameras oder Fotoapparate beispielweise und eine Praxis des Bildermachens. ${ }^{3}$ In beiden Fällen nehmen Beobachtende mit ihren jeweiligen Medien an Situationen teil, in denen sie forschen.

Ein Beispiel: Das Video Notieren und Verkörpern (Mohn, DVD 2011) ${ }^{4}$ befasst sich mit Praktiken und Medien der Rezeptionsforschung. Wie platzieren sich ethnographische Beobachterinnen gegenüber einem Publikum, das sie beforschen möchten? Was lässt sich von wo aus hören, sehen und notieren?

Abbildung 1: Schreibende Ethnographin
mitten im Kinderpublikum

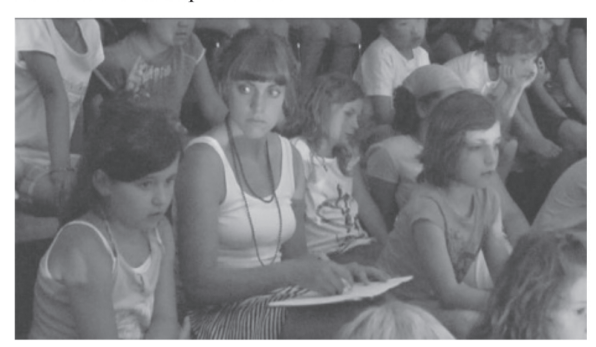

Abbildung 2: Ethnographin berichtet auf der Teamsitzung: „Sie haben immer „so“" gemacht!“"

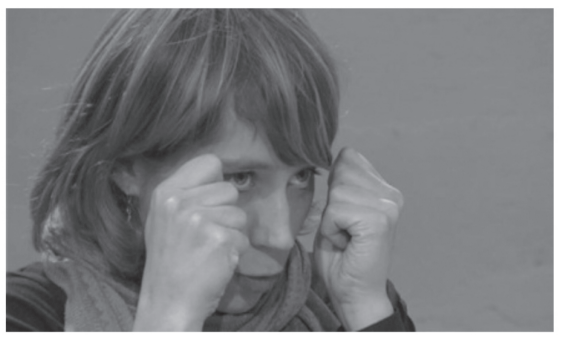

Quelle: DVD Mohn (2011)

3 In seiner foto-ethnografischen Studie „Strassenräume. Berlin, Shanghai, Tokyo, Zürich“ schreibt Krusche: „Mohn arbeitet mit Video, doch viele der Überlegungen und Erkenntnisse treffen auch auf die Fotografie zu, weshalb die Arbeit mit der Fotokamera unter Berücksichtigung gewisser Anpassungen als eine Variante der Kamera-Ethnografie angesehen werden kann“ (Krusche / Vogt 2011: 20). Die Grundüberlegungen der Kamera-Ethnographie sind auf diverse Medien der Ethnographie (nicht zuletzt auch auf den Umgang mit Schreibzeug) übertragbar. Eine Audio-Ethnographie beispielsweise operiert über Lauschpfade, führt akustische Erkundungen durch und zielt auf ein dichtes Hören komplexer Geräuschkulissen. In diesem Text geht es mir vorrangig ums Zeigen. Daher konzentriere ich mich auf Bildgebungsprozesse und den Gebrauch der Kamera beim ethnographischen Forschen.

4 Produziert im Rahmen des Projektes „Ästhetische Kommunikation im Kindertheater“ an der Zürcher Hochschule der Künste, 2009-2011. 
Offenbar hat sich die schreibende Forscherin mit dem Ziel, Äußerungen der Kinder beim Zuschauen zu erheischen, ganz anders positioniert als die Kamerafrau (siehe Perspektive der Kamera) in ihrem Wunsch, Gesichter von vorne sehen zu können. Was macht das Schreiben mit einer Beobachtung, was das Filmen? Schreibakte haben eine spezifische Dauer, die das Beobachten strukturiert: Der Kopf der Beobachterin hebt sich beim Gucken, senkt sich beim Schreiben; gelegentlich parken ihre Blicke nach vorne zur Bühne gerichtet, bis ihre Augen wieder hin- und herschwenken und eine ganz andere „Aufführung“ fokussieren: Kinder als Theaterrezipienten. Auch die Körper der Forschenden spielen als Darstellungsinstrumente im Beobachtungsprozess eine wichtige Rolle: „Die Kinder haben während der Aufführung immer so gemacht", berichtet eine der Forscherinnen während der Teamsitzung. Wo die Grenze des Sagbaren erreicht ist, markiert typischerweise das Wörtchen „so“ den Einsatzpunkt von Körperdarstellungen.

Die übliche Unterscheidung in „Teilnehmende Beobachtung“ hier und „Videographie“ dort versäumt die Reflektion der jeweiligen Medien. Wir haben es z.B. mit schreibenden versus filmenden Beobachtungen zu tun; mit spezifischen Instrumentarien und Fertigkeiten des Beobachtens. Der Videographie-Begriff suggeriert, dass Ethnographie mit der Videokamera betrieben wird, tatsächlich fällt er aber meist im Zusammenhang von visueller Datenerhebung, Videomitschnitt oder videogestützter Ethnographie und spiegelt dabei die Trennung in einen Begriff von „Ethnographie“, der das Schreiben nicht als medialen Aspekt der Wissensgenerierung reflektiert, und in einen Datenbegriff, der wiederum als von der eigentlichen Ethnographie getrennt konzipiert wird.

Unter dem Dach der Videographie haben sich spannende Ansätze im Einsatz audiovisueller Medien entwickelt und einige davon bestimmen ihre Nähe zur Ethnographie explizit. Knoblauch, Schnettler und Raab z.B. argumentieren für den Videographie-Begriff so:

„, The importance of background knowledge that elucidates visual aspects of the recordings proves again the importance of ethnography for doing video-analysis. [...] The task of the researcher is to acquire sufficient knowledge, particularly those elements of knowledge, partly embodied, relevant to the activity on which the study focuses. [...] It is due to this close relationship between video analysis and ethnography that I suggest the term videography adequately accounts and describes the unified core of what constitutes the interpretative video analysis methodology. " $(2012: 79,81)$

"It is, by the way, this orientation towards "natural situations" that leads video analysts to sympathise strongly with ethnography, particular with the kind of ethnography which turns towards encounters, social situations and performances as championed by Erving Goffman (1961, 1967, 1971)." (Knoblauch / Schnettler / Raab 2012: 11)

Demnach herrscht eine Sympathiebeziehung zur Ethnographie, eine Freundschaft, die in einer ethnographisch orientierten Analyse natürlicher Videodaten zum Ausdruck kommt. Mit der Kamera-Ethnographie folge ich einem Forschungsverständnis, das auf den Entwurf ethnographisch beobachtender Blicke, Bilder und Bildfolgen zielt und sich als medienreflexive Ethnographie versteht. Kamera-Ethnographie ist unter ethnographischer Adresse angesiedelt, im Bezirk praxeologisch orientierter Ethnographie. 
Beobachten schreibende Ethnographen alltägliche Praxis, so stehen sie vor einem Problem, das Hirschauer (2001) die „Schweigsamkeit des Sozialen“"5 nennt. Nun sind Phänomene des Sozialen keineswegs schweigsam: Es wird geredet, gelacht, geräuspert, gesungen. Doch etwas gibt es tatsächlich, was sich nicht einfach von einem laufenden Tonband einfangen lässt, nämlich das, was dichte Beschreibung (Geertz 1987[1983]) zum Gegenstand hat. Ethnographie, so Hirschauer, stehe vor der Aufgabe, etwas zu formulieren, was zuvor noch nicht in Worte gefasst war. Situationen können nicht ,abgeschrieben“ werden, sondern sie erfordern stets eine Methodik und Praxis der Hervorbringung von Beschreibung.

In ihren Grundzügen ist die Kamera-Ethnographie zunächst aus einer Übersetzung des soziologisch-praxistheoretischen Ethnographie-Konzepts (Amann / Hirschauer 1997) ${ }^{6}$ in ein audio-visuelles Medium entstanden. Von wo aus blicke ich wohin? Wo positioniere ich mich in diesem Raum und wie meine Forschung in diesem Feld? Zu beobachten erfordert Blicke und Perspektiven, mit denen man dies tun kann.

„, ,Sehen' zum Beispiel ist kein passiver Vorgang, durch den bedeutungslose Eindrücke gesammelt werden, damit der ordnende Geist aus diesen amorphen Daten Formen für seine eigenen Zwecke herauslesen kann, ,Sehen 'ist selber schon ein Formulierungsprozess; unser Verständnis der sichtbaren Welt beginnt im Auge" (Langer 1984: 97).

In Analogie zur „Schweigsamkeit des Sozialen“ stellt sich den Kamera-Ethnograph/innen das methodische Problem einer „Bildlosigkeit des Sozialen“. Auch hier gilt, dass Phänomene des Sozialen keineswegs unsichtbar sind: Es wird gestikuliert, geblickt, getanzt, geräumt. Natürlich auch erkennbar geredet. Um solches Geschehen in Bilder und Bildfolgen zu transformieren, bedarf es jedoch einer medialen Praxis, die kein Abfilmen ist, sondern ein Entwurfsprozess der Verbildlichung. Erst über eigene Blicke entstehen gestaltete Bilder, die den weiteren Forschungsprozess an den Bildern und mit den Bildern ermöglichen.

Verbildlichung ist die Voraussetzung für Anschaulichkeit, die erst ein Zeigen / Präsentieren situierter Beobachtungserfahrungen ermöglicht. Die Differenz des Visuellen kann verortet werden in der Eigenschaft von Phänomenen des Sozialen einerseits und dem konzeptuellen Ausgangspunkt der Forschungspraxis in der ethnographischen Feldsituation andererseits. In der visuellen Ethnographie herrscht allerlei Konfusion über die systematische Bedeutung dieser Differenzen für den Erkenntnisprozess. Gern werden sie ausgeblendet oder als technisch überwindbar betrachtet. Geertz spricht hingegen von einer „nicht zu umgehenden Tatsache, dass alle ethnographischen Beschreibungen hausgemacht sind, dass sie die Beschreibungen des Beschreibenden sind, nicht die der Beschriebenen“ (Geertz 1990: 139 f). Analog gilt für

5 „Es sind die Probleme des Stimmlosen, Stummen, Unaussprechlichen, Vorsprachlichen und Unbeschreiblichen, die das ethnographische Schreiben zuallererst zu lösen hat. In ihm wird etwas zur Sprache gebracht, das vorher nicht Sprache war. Für diese Aufgabe einer Verschiebung der Artikulationsgrenze muss sich die Beschreibung von der Logik der Aufzeichnung abwenden und zu einer theorieorientierten Forschungspraxis werden, die nicht nach ihrer Dokumentationsleistung, sondern nach ihren analytischen Leistungen zu bewerten ist [...] Nennen wir es die Schweigsamkeit des Sozialen“ (Hirschauer 2001: 429), schlägt Hirschauer vor. „Mit dieser Metapher ist nicht nur das bezeichnet, was die Alltagssprache unter Schweigen versteht - ein Aussetzen der Sprechpraxis - sondern zum einen eine Leerstelle für eine auf Verbaldaten fixierte Forschung, zum anderen eine stumme Herausforderung für Beschreibungen, etwas eben doch ,zum Sprechen zu bringen', das Widerstände gegen Verbalisierungen bietet." (Hirschauer 2001: 437).

6 Amann / Hirschauer formulieren ein soziologisches Ethnographiekonzept, das diese eigentlich der Ethnologie entlehnte Methode in das Licht einer empirischen Entdeckungsstrategie rückt, die alles andere als „bloß deskriptiv“ ist. Sie plädieren dafür, Wissensordnung des Feldes und Relevanzen der eigenen Disziplin auseinanderzuhalten und diese Unterscheidung konstruktiv zu nutzen bei der Hervorbringung feldbezogener Beschreibungen der Beschreibenden (vgl. Amann / Hirschauer 1997). Ähnlich argumentieren Mohn / Amann (1998: 4ff). 
eine ethnographische Blick- und Bildarbeit, dass hier Blicke und Bilder der Blickenden erarbeitet werden, ,hausgemachte“ wohlgemerkt.

Diese Einschätzung hat tiefgreifende Konsequenzen sowohl für die ethnographische Forschungsarbeit mit der Kamera als auch bei der weiteren Auseinandersetzung mit diesen (selbst) produzierten Bildern / Verbildlichungen.

\title{
3. Differenz der Blicke und Bilder
}

Die Kamera als einen Apparat des Blickens zu nutzen und Filmschnitt als Erkenntnisinstrument, dies ist gegenüber Versuchen möglichst blickfreier Datenaufzeichnung oder der Verfilmung von Drehbuchinhalten wenig verbreitet. Mit der Kamera-Ethnographie wird an dieser Stelle eine eigenständige Strategie verfolgt.

In Situationen zu beobachten stellt vor ein Problem: Soziale Situationen lassen sich nicht bei laufender Kamera aufzeichnen. Es gibt keinen fixen Punkt, von dem aus alle Aspekte des sich entwickelnden Geschehens erfasst werden könnten. ${ }^{7}$ Es ist daher auch gar nicht möglich, mit einer Kamera Zeiten (z.B. Schulstunden), Orte (z.B. Kindertagesstätten) oder Personen (z.B. Kinder) zu „videographieren“. Dass die Abbildbarkeit sozialer Situationen praktisch dennoch vielfach unterstellt wird, hängt mit den Erfordernissen anderer Forschungsanliegen zusammen, die am technisch generierten, zusammenhängenden und in Zeitlupe wiederholbaren dokumentarischen Material interessiert sind, das wie ein „Materialkörper“ anatomisch seziert und sequenzanalytisch aufgeschlüsselt werden kann. Mondada bringt dies prägnant zum Ausdruck, wenn sie Anforderungen an einen ethnomethodologisch-konversationsanalytischen Videogebrauch benennt:

\begin{abstract}
„The ethnomethodological conversational inquiry adopts specific ways of shooting video which preserve key dimensions such as: time. Openings as well as closings of an activity, its particular length and rhythm are respected: one continuous shot documenting the whole activity is the specific video response to this feature [...] participation framework and interactional space. All relevant participants are considered: this produces video shots avoiding focussing too narrowly on only one participant [...]. This requirement implies a way of monitoring not only the ongoing action but all possible participants attending to it and produces a strong sense of the complexity of the interactional space-documented either with a static camera (allowing the cameraman to be absent, but then having to anticipate all possible movements done by the participants) or with a mobile camera (imposing the presence of the cameraman, who locally accompanies and projects the next possible action)." (Mondada 2012: 54f)
\end{abstract}

Forschungsmethoden üben Handhabungszwänge im Mediengebrauch aus. Was einer ethnomethodologisch ausgerichteten Konversationsanalyse dient, taugt für die Realisierung ethnographisch beschreibender / zeigender Entwurfsprozesse nicht. Und umgekehrt gilt dasselbe: Eine Ethnographie mit der Kamera liefert Verbildlichungsentwürfe anstelle der für andere Zwecke erforderlichen Datengrundlage. ${ }^{8}$

7 Als filmende Beobachterin kann ich mein Augenmerk auf Körperkonstellationen im Raum richten. Dies erfordert genügend Abstand und evtl. einen Blick von der Seite auf die miteinander Interagierenden. Interessieren mich jedoch Mimik und Gestik, so werde ich mich an andere Stellen im Raum begeben. Möglicherweise ist das Hantieren mit Gegenständen auf einer Tischfläche so interessant, dass ich mich, Kamera drehbereit, mit an den Tisch setze, Rücken zum Fenster (Gegenlicht vermeiden), frontal zu mindestens einem der Interaktionspartner (Mimik sehen können) und so, dass weitere Personen per Kameraschwenk fokussiert werden können.

8 Streeck (2007) zeigt jedoch, wie sich gerade an kamera-ethnographisch fokussierten Videosequenzen überraschende Mikro-Analysen von Interaktion durchführen lassen. 
Filmaufzeichnungen, bei denen möglichst alle beteiligten Körper und Objekte ständig zu sehen sind, mithilfe von Kamera-Automaten durchzuführen ist in meinen Augen nichts Harmloses: $\mathrm{Zu}$ keiner Beobachtungsbeziehung fähig wird eine ununterbrochen laufende Kamera zum gnadenlosen Gegenüber; die Regisseur/innen des Mitschnitts neigen dazu, den Raum zu verlassen oder spielen ,toter Mann“. So wird in der Absicht, Eingriffe in die beobachtete Situation zu vermeiden, Datenerhebung durchaus penetrant und die Beobachteten bleiben der Kamera ausgeliefert. Nicht selten führen spätere Versuche der Anonymisierung solcher Daten zu schwarzen Balken auf Gesichtern von Erwachsenen und Kindern; Forschungsfelder erscheinen plötzlich als Deliktzonen; eine Scham der Forschenden macht Forschung unverschämt (vgl. Mohn 2012: 200). Eine interagierende und reagierende Kamera-Beobachtung löst diese Problematik durch Präsenz, da Ethnograph/innen wahrnehmen und empfinden können; sich bewegen und ihre Position verändern; soziale Räume, in denen geforscht wird, unverhohlen betreten und auf das Sehen und Gesehen werden achten. Sie sind kontinuierlich damit befasst, Beobachtungsbeziehungen aufzubauen und zu erhalten. Das Natürliche an einer „,natürlichen Situation“ besteht im Zusammenspiel aller an ihrer Durchführung und Beforschung hier und jetzt Beteiligten. Auch Befürworter/innen natürlicher Situationsdokumente reflektieren dies:

„Videos produced within the naturalistic requirements of ethnomethodology and conversation analysis both aim at preserving relevant details and phenomenal field features and reflexively contribute to the configuration of the very interactional order they document."

(Mondada 2012: 64)

Ethnographie zu betreiben bedeutet, die Tatsache situierter Praxis offensiv zu nutzen. An den Grenzen zur Taktlosigkeit, durch die Kamera noch verschärft, zeigt sich eine eigenartige Situationsteilnahme der Ethnograph/innen: Sie praktizieren eine Art professioneller Ignoranz bei gleichzeitiger Aufmerksamkeit. Ihr Hinschauen gelingt im Weggucken. So folgen Ethnograph/ innen mit der Kamera evtl. den Blicken der Teilnehmenden gerade nicht, sondern beobachten stattdessen in aller Ruhe, wie deren Blicke wandern. Filmsequenzen beendet man besser genau dann noch nicht, wenn Sprechende ihren Punkt setzten. Es wird sozusagen immer ein wenig über die Ethnomethoden des Feldes hinaus gefilmt, um sie sich anschauen zu können. Reaktiv zu sein bedeutet für Kamera-Ethnograph/innen keinesfalls, auf alles und jedes so zu reagieren, wie es andere Teilnehmer/innen auch tun würden. Ethnograph/innen sind nicht allein in der Rolle der Offenen, Wahrnehmenden und Sensiblen, sondern auch in der Nähe ,verrückter“ Interaktionsteilnehmer. Eine Rollendifferenz bleibt zu kommunizieren, in der sich Beobachtende verständlicher Weise ,anders“ verhalten. Solche Differenzen können nur in der jeweiligen Situation taktvoll ausbalanciert werden (vgl. Mohn 2012: 202). Die Beobachtbarkeit des Sozialen ist per se doppelt konstituiert: als eine lokal hervorgebrachte Praxis der beobachteten Akteure und als eine der situierten Beobachtung.

Woher nehmen Ethnograph/innen ihre Selektionskriterien? Wie entstehen ethnographische Blicke und Bilder der Forschenden? Vorab? In der Situation? Nach Plan? Improvisiert? Aus dem Bauch? Aus Versehen? Aus dem Theorieladen? Blicke entstehen zu lassen und zu entwerfen, die die Forschung voranbringen, kann Ergebnis tagelanger Teilnahme im Feld und stundenlanger Sichtung erster Materialien und Skizzen aus dem Feld sein. Mögliche Fokussierungen, die beim nächsten Feldaufenthalt das beobachtende Filmen ausrichten, entwickeln sich im Rahmen zirkulärer Forschungsprozesse zwischen den Feldaufenthalten, beim Interpretieren der Materialien und beim Aufwerfen neuer Forschungsfragen. Doch auch in der Beobachtungssituation selbst werden Blickschneisen spontan entworfen und variiert, manchmal zunächst intuitiv oder in Form einer mit dem Geschehen mitschwingenden „Ciné Trance“. Es sind Versuche, ein komplexes Geschehen selektiv handhabbar zu machen (vgl. Mohn $2008 \mathrm{a}$, 2010). Die persönliche Blicksuche der Ethnograph/innen ist eingebunden in die Denk- und 
Blickstile ihrer wissenschaftlichen Kollektive (vgl. Fleck 1983 / 1936). Drei kurze Beispiele (DVD Mohn / Amann 2006): ${ }^{9}$

Während auf der Ton-Spur Schreibutensilien rascheln, gelegentlich von einer Anweisung der Lehrerin übertönt, die das Schreiben eines Physik-Tests überwacht, zeigt die Bild-Spur des Videomaterials jugendliche Schüler/innen beim Test-Schreiben und befragt diese ganz auf individuelle Leistung zugeschnittene Situation daraufhin, wie der Lernkörper sie vereinzelt und als Ensemble meistert: Entdeckt wird dabei ein „Blickwandern“ als stumme Form subtiler Mikro-Interaktion.

Während sich auf der akustischen Seite des Geschehens Fragen einer Englischlehrerin mit Antworten von Schüler/innen abwechseln, wird auf der Bild-Ebene genau nicht dieses PingPong verfolgt. Immer wieder verweilt der Blick der Kamera bei Armen und Händen auch derjenigen, die sich gemeldet haben und nicht dran gekommen sind. Sichtbar wird eine sonderbare Praxis der Jugendlichen, die sich im Luftraum des Klassenzimmers abspielt und dort wohl mit Sichtbarkeit rechnen kann.

Während in einer Freiarbeitsstunde das auf die Kamera montierte Richt-Mikrophon allgemeines Gemurmel und gelegentliche Gesprächsfetzen aus den zur Kamera nächstgelegenen Reihen des Klassenzimmers verzeichnet, bedienen die gewählten Bildausschnitte die Frage: Wie gehen die jugendlichen Schüler/innen damit um, bei dieser Unterrichtsform aufstehen zu dürfen, um sich Arbeitsblätter und Bücher zu holen? Die Kamera verfolgt Bewegung im Raum, die Gänge im Klassenzimmer. Am Schneidetisch wird später sichtbar, dass die Wechsel vom Aufstehen, Gehen und Hinsetzen einer unausgesprochenen Choreographie zu folgen scheinen, denn nie laufen alle auf einmal und niemals läuft niemand. Der Lernkörper hält in der Freiarbeitsstunde ein Perpetuum Mobile am Laufen!

Abbildung 3

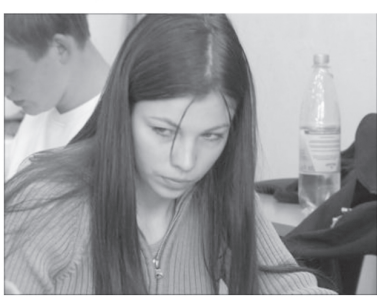

Abbildung 4

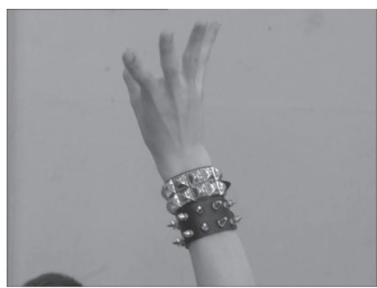

Abbildung 5

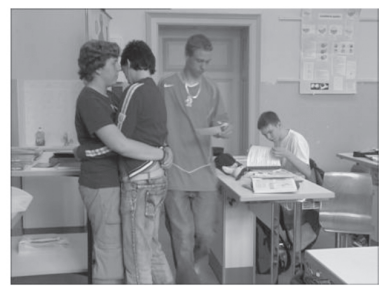

Quelle: DVD Mohn / Amann (2006)

Die Medienspezifik dieser Beispiele liegt auf der Hand: Ob es die Organisation der Blicke, die Varianz der Meldegesten oder die Choreographie bewegter Körper im Raum ist, allen Themen ist gemeinsam, dass sie weder mit einem Tonband noch über eine Transkription des

9 Video „Stundenweise Schulzeit“ (Mohn), auf: DVD Lernkörper. Kamera-Ethnographische Studien zum Schülerjob. 
Gesprochenen einer Beforschung zugänglich wären. ${ }^{10}$ Es sind Kamera-Themen (vgl. Mohn 2012: 208). Zugleich beruhen diese Beispiele auf dezidiert ethnographischer Methodologie. Sie haben gemeinsam, dass bereits im Umgang mit der Kamera - in der sogenannten Datenerhebungsphase - Fokussierungsentscheidungen getroffen wurden, die im weiteren Prozess ergiebig waren, sich verstärken und verdichten ließen und bei denen Aspekte des Feldes und der Forschung schließlich aneinander zur Darstellung kommen (vgl. Mohn 2002, 2008 b).

Das „Blickwandern“ der Schüler/innen im obigen Beispiel wurde für die filmende Ethnographin in einer Situation wahrnehmbar, wo weder gesprochen noch herumgelaufen werden durfte: Physiktest. Eine Kamera kann nicht in die Köpfe schauen, sie bleibt beim Körper außen vor, reduziert auf das, was sich zeigt. ${ }^{11}$ Die in der Untersuchungssituation gebotene Stille und das Stillsitzen erzeugen nun auch für die Kamera-Beobachtung ein minimalistisches Untersuchungsdesign, wo es schließlich die Blicke sind, die sprechen und den Raum zu durchstreifen scheinen. Hier zeigt sich ein Zusammenspiel von Situation, Medium, ethnographischer Methodologie und Diskurs. Auf der Suche nach Interaktion und Bewegung, Körpersprache und Jugendkultur im Unterricht, nach sichtbaren Äußerungen der nun auf sich allein gestellten Individuen, spürt die Kamera subtile Aspekte auf, wirft den Blick auf gesenkte Köpfe und betrachtet das, was dabei zu betrachten übrig bleibt: etwa zu Igelstacheln gefestigte Frisuren, über die auch die Blicke der Lehrerin schweifen könnten und die etwas von der Person zeigen, während ein Schüler verborgen seine Leistung absolviert. Dass hier die Blicke interessieren, wundert kaum: Sie sind wie Fenster in geschlossenen Häusern, mal mit Vorhängen aus Haaren zugezogen, mal etwas, mal ganz weit geöffnet. Und was da wie hinaus sieht, löst im Moment des Filmens nur fragendes Hinschauen und eine Konzentration der Kamera-Einstellung aus benannt werden kann es (noch) nicht (vgl. Mohn 2010: 211).

Das Hinschauen gelingt aus dem Impuls, sich für etwas zu interessieren, den Dingen nachzuspüren, sie zu befragen. Auf professionelle Weise werden Wissen und Nicht-Wissen in einer Schwebe gehalten, kann dabei etwas in den Blick geraten und in den Blick genommen werden. Diesem sich erst noch formenden Interesse ,an etwas“ verleihen die gewählten Kamera-Einstellungen Gestalt. Die Kamera wird dabei zur Caméra Stylo („Kamera als Federhalter“, Astruc 1964), zu einem Instrument des Formulierens audiovisueller Beobachtungsnotizen, die zugleich etwas über die Anwesenheit und Blicksuche der Ethnograph/innen im Feld zeigen und etwas über Aspekte des beobachteten Geschehens. So entsteht ein hybrider Datentypus, bei dem Feld und Forschung nicht ohne einander zu ihrer Darstellung kommen.

Die durch einen blickenden Kameragebrauch geleistete „Thematisierung“ des Beobachteten ist nicht im verbalen Sinne zu verstehen. Es findet eine Fokussierung, ,auf etwas“ statt. Dieses „Etwas“ bleibt unbestimmt, ist aber Gegenstand eines gerichteten Interesses beim Filmen, durch das es schließlich - auch sprachlich - bearbeitet werden kann. Frames werden zum

10 Auch der Versuch komplexerer Notationsverfahren, die sich um die Integration visueller Aspekte bemühen, nutzt einer zeigenden Ethnographie wenig. Die Idee der „Feldpartitur“ (entwickelt durch C. Moritz) zeigt, pro Din-A4 Seite können zwei Sekunden Video in einem kunstvollen Transcript veranschaulicht werden, das einer Orchesterpartitur ähnelt. Moritz hat die „Feldpartitur“ als ,,mikroprozessuale Transkription von Videodaten über einer Zeitachse“ entworfen. „Die Videotranskription der Feldpartitur ermöglicht es, naturgemäß flüchtige Videodaten innerhalb eines schriftsprachlichen Mediums zu fixieren“(Moritz 2009). Die Arbeit daran scheint mir die Zeit zu verschlingen, die ein Forschungsprojekt zur Erkundung des Feldes zur Verfügung hätte und die komplexe Partitur schließlich zu lesen, führt nicht unbedingt zu interessanteren Ergebnissen als die Betrachtung der Bildsequenzen vor ihrer aufwendigen Transkription. Was also tun? Kamera-Ethnographie bedeutet, mit dem audiovisuellen Material selbst weiterzuarbeiten.

11 Sozialtheoretisch betrachtet gilt dies ebenso für das, was die beobachteten Akteure selbst beobachten können. Der „,verzweifelt suchende Blick“ eines Schülers zeigt, was er für den Mitschüler bedeutet: „Hast du eine Antwort auf die Frage, die wir hier im Test beantworten sollen?“. 
Terrain des Fragens. Die performative Qualität und ästhetische Attraktivität solchen Bildmaterials ergibt sich im Gelingen des Versuchs, Konzentration auf das zu Bestimmende zu erzeugen und die Wahrnehmbarkeit von Aspekten der Untersuchung zu provozieren. ${ }^{12}$ Über den Entwurf und die Variation von Blickschneisen lässt sich eine anhaltende Erneuerung ethnographischen „Befremdens“ realisieren (vgl. Amann / Hirschauer 1997). Wie landet dann aber „die Situation“ im Datenmaterial? Gar nicht. Das sogenannte „Ganze“ wird zerlegt in Teilaspekte, die sehr viel später erst in mögliche Zusammenhänge gebracht werden.

So ermöglicht der jeweilige Datenbegriff unterschiedliche daran anknüpfende Forschungsschritte: An „natürlichen Daten“, die jedoch selbst nicht schon als ethnographisch gerahmt sein dürfen, lassen sich ethnographisch informierte Analysen und Rekonstruktionen durchführen und abschließen. Im hybriden Datenmaterial lässt sich der ethnographische Prozess selbst sukzessive materialisieren und entfalten, was auf die Kommunikation ethnographischer Erfahrung und Analysen in fortgesetzten Prozessen des Sehens und Zeigens zielt. Es ist nur konsequent, wenn beide Richtungen zur Lösung praktischer Probleme beim Forschen jeweils dort nach Anregungen Ausschau halten, wo bereits Expertise dazu vorliegt, z.B. bei Praktikern des Umgangs mit den Aufzeichnungen von Überwachungskameras ${ }^{13}$ oder im anderen Fall etwa bei Expert/innen aus Medienkunst und Experimentalfilm, Öffentlichkeit und Erfahrung.

Gerade weil sie keine Situationskopien sind, können kamera-ethnographische Bildsequenzen auf Resultate zielen, die produktive Differenzen beanspruchen: zu den real verlaufenden Situationen, in denen sie entstehen; zu dem, was Situationsteilnehmer/innen erzählen können; zu dem, was die Forschenden selbst zuvor wussten. Wenn wir die Blickdifferenz beim Filmen zum Verschwinden bringen würden, etwa indem wir audiovisuellen Aufzeichnungen einen Dokumentcharakter natürlicher Daten unterstellen, dann schwindet zugleich diese genuine ethnographische Erkenntnischance situierter Beobachtung. Ziel ist es, über die Generierung von Bildern und Bildfolgen das Sehen und Verstehen voranzubringen und ein kommunikatives Zeigen ethnographischer Erfahrung möglich zu machen.

\section{Differenz des Gegenstandes}

Schließlich soll es darum gehen, etwas zeigen zu können. Medien der Ethnographie konstituieren ihre Themen, ein Beispiel aus den 1980er und 1990er Jahren veranschaulicht dies bilderbuchhaft: Bei seinen Forschungen im Biologielabor entdeckte Latour schreibend Biologen als ,manic writers" und fügt hinzu: "Activity in the laboratory had the effect of transforming statements from one type to another" (Latour 1979: 48, 81); mit einem Tonband ausgerüstet wurden wenige Jahre später Amann und Knorr Cetina auf die Geschwätzigkeit von Laborkultur aufmerksam und stoßen auf ein ,in extremer Weise mündlich strukturiertes Labor" und die Zeit, die in bestimmten Laboratorien auf Gespräche verwendet würde mache ein Vielfaches der Schreibtätigkeit aus, von der Geertz (1973) und Latour / Woolgar (1979) berichten, so

12 Häufig bietet die ästhetische Qualität von Videobildern Anlass zu Grenzziehungen zwischen Wissenschaft und Kunst. In der Writing-Culture-Debatte (vgl. Clifford / Marcus 1986; Berg / Fuchs 1993) wurde von „Poetics of Ethnography“ gesprochen. Die Rolle von Ästhetik und Poesie scheint mir jedoch gerade im Hinblick auf ethnographische Forschungsprozesse noch wenig geklärt.

13 Heath schreibt: "Individuals who use surveillance equipment [...] are themselves sensitive to the views on the world beyond the image, and orient to the relationship between their view and the domain itself. [...] It also happens that these methodological and analytic sensitivities inform the analysis undertaken in this paper. As Sacks suggests when faced with complex methodological problems, an investigation of practioners who have similar concerns and problems in their occupational or professional life, might suggest ways of overcoming these problems. When tackling contemporary problems in the analysis of video materials we should not overlook the skills of and practices of those who, in the course of their everyday work, collect video data, analyse these materials and present and discuss them with others." (Heath 2012: 46, 48). 
Knorr Cetina (1988: 94); wen wundert es, wenn in den 1990er Jahren die mit einer Kamera ausgerüstete Studentin im Labor in ihr Tagebuch schreibt:

„Biologen verbringen den größten Teil ihrer Zeit damit, Sichtbarkeit herzustellen und betreiben eine besondere Repräsentationskultur. Sie benutzen Filme, um darauf erstmalig Spuren ihres Untersuchungsgegenstandes zu sehen. Embryos werden in Bilder umgestaltet, die gleichzeitig Organismus und Repräsentation sind. "(Mohn 8.5.1992)

\section{Abbildung 6: Rätselhafte Zeichen im Bildausschnitt der Kamera}

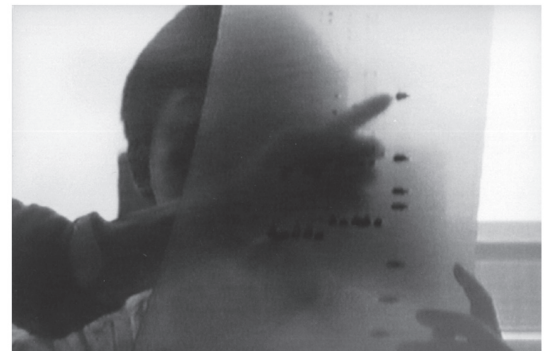

Im Rahmen der Kamera-Ethnographie wurden bislang insbesondere Praktiken beforscht, die sich einer an Sprache und Text orientierten Forschung entziehen, es sind dennoch klassische Themen der Ethnomethodologie (vgl. Weingarten / Sack 1979[1976]): die Wieund Wann-Fragen alltäglichen Handelns; Choreographien und Rhythmen; Körperlichkeit und Objekte; Interaktion, Bewegung, Gestik und Mimik; stumme Praktiken und sogar Praktiken des Passiven. ${ }^{14}$

Quelle: Mohn (1993)

So führen beispielsweise die bereits zitierten Meldegesten der Jugendlichen im Unterricht eine Praxis neben der Sprache vor, während uns die Beobachtung unter 3-jähriger Kinder beim Mittagessen in der Kita mit Vorsprachlichkeit konfrontiert. Die Kinder können noch gar nicht sprechen: Sie klopfen mit den Löffeln, summen in die Tassen, werfen ihre Arme in die Luft oder ahmen den Klang von Worten nach. Ihr „Tischgespräch“ fällt weitgehend rhythmisch musikalisch aus. Die akustische Seite des audiovisuellen Mediums rückt ins Zentrum der Analyse und auf der Video-DVD wird schließlich das „Rhythmische Ensemble“ als Film und reines Hörstück angeboten. ${ }^{15}$

Neben dem medialen Aspekt ist für das Vorhaben einer zeigenden Ethnographie der methodologische Aspekt von Belang. In der These von der „Schweigsamkeit" und „Bildlosigkeit“ des Sozialen ist die Spur bereits angelegt, die sich auch in der Bearbeitung visuellen Materials fortsetzt. Während beim beobachtenden Filmen das Erproben von „Blickschneisen“ von Kamera-Ethnograph/innen die Gestaltung von Beobachtungsbeziehungen erfordert - Improvisationstalent und ästhetisches Gespür, Empathie und Reflexivität, persönliche Präsenz und Interaktionsfähigkeit -, spielt beim Sichten, Benennen, Zerlegen, Thematisieren und Montieren audiovisueller Sequenzen im Visualisierungslabor auch kollektive Kreativität und Theoriearbeit eine Rolle. Neben die „Blickschneisen“, die beim Filmen das Material an den Schnittstellen von Feld und Forschung strukturiert haben, tritt nun das analytische Potenzial des Filmschnitts und somit eine weitere, tatsächliche Schnittstelle: Beim Auftrennen des audiovisuellen Materials in Sequenzen hängen die Relevanzen der Sequenzierung ebenso von den jeweiligen Forschungsfragen ab wie von den Handlungssequenzen, die sich vor der Kamera

14 Bislang sind diese kamera-ethnographischen Beiträge zur Erforschung stummer Praxis publiziert: in Mohn / Amann (2006) Videos „Standby“, „Takt“, „Taktik“ und „Stundenweise Schulzeit"; in Mohn / Hebenstreit-Müller (2007) Videos „Zeit zum Gucken“, „Kinder als Beobachter“, „Erzieher als Beobachter“ und „Jara und Romy“; in Mohn / Wartemann (2009) Videos „Kinderpublikum“, „Erwachsene Assistenten“, „Blicke des Performers“, „Krisenexperiment“ u.a.; in Mohn / Wartemann (2011) Videos „Notieren und Verkörpern“ und ,Zuschauen Hand haben“; in Mohn / Breidenstein (2013) Videos „Temporärer Körperraum“ und „Ambulante Interaktion“.

15 Vgl. Buchholz (2005): Die Geburt der Sprache aus dem Geist der Musik. 
abgespielt haben. Wie arrangiert und von wo aus befragt antwortet das Material wie? Solche Versuche am Material können auch lehrreich scheitern. Diese Phase des Experimentierens und der Einbeziehung des Forschungskollektivs zielt noch nicht darauf, das Material in einen Film zu verwandeln - in welchen auch, ist doch immer noch ungeklärt, was hier überhaupt zu sehen sein könnte! Die späteren audiovisuellen Produkte profitieren jedoch von diesen Versuchen, bei denen sich Selektionsentscheidungen bei der Kameraführung anhand der Selektivität beim fokussierenden Schneiden zu potenzieren scheinen. Nicht wenige solcher Versuchsanordnungen des Materials wurden bislang Grundlage der publizierten Endfassungen. Hierin sehe ich einen kamera-ethnographischen Beitrag zum Genre ethnographischer Forschungsfilme.

Wir können nicht davon ausgehen, dass das, was wir beforschen wollen, am oder im Material bereits sichtbar und beobachtbar ist. Kamera-Ethnographie bedarf einer experimentellen Praxis. An Schreib- und Schneidetischen geht es nicht allein um Auswertungen des vorhandenen Materials, so wie es ist, sondern zuallererst um den Versuch, Beobachtbarkeit am und im Material zu erzeugen (vgl. Amann / Mohn 1998). Für dieses Vorhaben ist audiovisuelles Material bestens geeignet, denn neben seiner Wiederholbarkeit und Manipulierbarkeit im zeitlichen Ablauf kann es auf unterschiedliche Weisen zerlegt und bearbeitet werden. Das begleitende Schreiben gehört ebenso dazu wie ein differenzierendes, fokussierendes Schneiden und das Erproben interpretativer Rahmen, passender Kategorien und hilfreicher Theoriebezüge.

Es sind nicht allein die durch die Kameraführung zum Thema gemachten Praktiken und Phänomene, die ohne Worte daherkommen, auch den Forschenden bleiben angesichts des Videomaterials zunächst die Worte aus: Das Schweigen der Praxis - und nun des Materials erzeugt eine Leerstelle, der es bedarf, um begrifflichen Zugänge zu entwickeln. Auf der Suche nach Worten kommen Metaphern ins Spiel und verwickeln die Bildsequenzen in Wortspiele. Betrachten wir es doch mal ,als ...". ${ }^{16}$ Die Poesie der Praxis wird in ihrer Eigenartigkeit und Unbestimmtheit umschrieben, dies alles mit dem Ziel, aus dem mithilfe der Kamera geleisteten Hinschauen Sehen zu machen. „Schauen, Sehen, Wissen“ (Fleck 1947[1983]) funktioniert anhand bekannter Gestalten. Die Beschreibung nonverbaler Praktiken bedarf der Metapher als einem „Kunstgriff des Umverstehens“ (Blumenberg 2001: 194).

Dabei können Metaphern das Gesehene keinesfalls adäquat bezeichnen und sind alles andere als eine Kopie der Bilder in Wortform. Doch kommen an den Bildfolgen die hausgemachten Blicke und ersten Beschreibungsversuche allmählich ,,auf Kurs“. Ein Beispiel für die zirkuläre Entfaltung dichten Zeigens: Die experimentell angelegte Collage „Standby“ (Mohn, in: DVD Mohn / Amann 2006) interessiert sich für Körperhaltungen, in denen Jugendliche im Frontalunterricht Ruhezustände zeigen, die bis zur scheinbaren Leblosigkeit reichen.

16 Buchholz (1999: 90) widmet dem „als“ einen eigenen Abschnitt und schreibt darin: „Ich betone das Wörtchen , als" deshalb, weil daran erkennbar wird, dass hier jeweils sehr großformatige metaphorische Resonanzen gebildet werden. Dass es sich um Metaphern handelt, wird nur dann erkennbar, wenn man das Wörtchen ,als` stehen lässt. Wird es beseitigt, dann werden aus Metaphern wissenschaftlich scheinende Definitionen.“. 
Abbildung 7: „Standby-Modus im Frontalunterricht

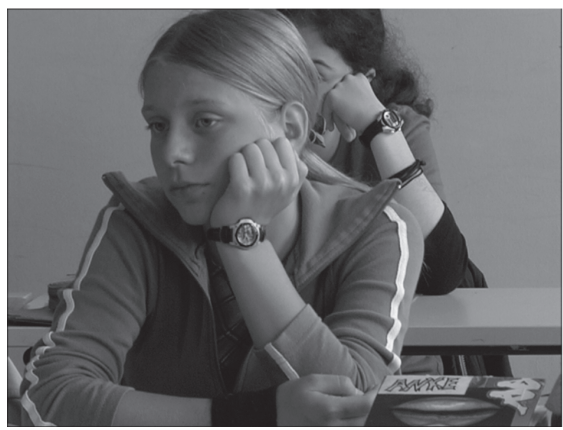

Abbildung 8: „Standby“ und „Blickfernbedienung“

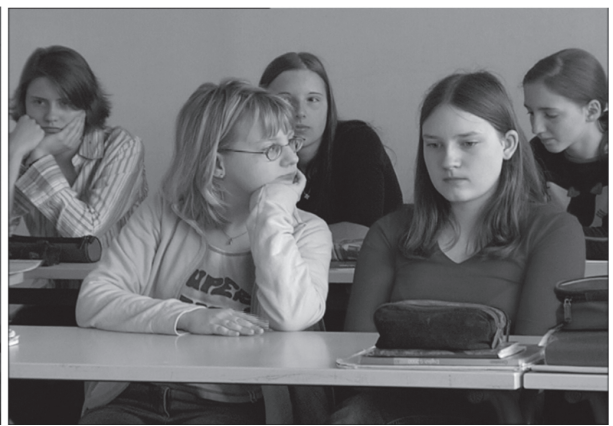

Quelle: DVD Mohn / Amann (2006)

Die Vorgeschichte dieses Videos beruht auf dem Scheitern einer Versuchsanordnung zum Thema Langeweile. Zitat aus dem Forschungstagebuch:

„,Wie genau äußert sich Langeweile? Ich spiele das Videomaterial ab und teste meine eigenen Differenzierungskriterien: Langeweile - keine Langeweile. Meine Erwartung, dass ich nun darauf stoße, was Langeweile alles sein kann, wird enttäuscht. Bei jedem genauen Hinsehen entwischt sie mir, die Langeweile. Sie entgleitet in ein anderes, ein benachbartes Phänomen, das Langeweile allein berührt, aber nicht darzustellen vermag, wie z.B. Müdigkeit oder Apathie, Unruhe oder abgelenkt Sein. Es entsteht ein Liste von Videosequenzen, die Langeweile nicht zeigen und eine Liste von Begriffen, die Langeweile wie ein schwarzes Loch umtanzen. " (Mohn, 31.1.2003)

Da eine Videobeobachtung angewiesen ist auf das, was sich zeigt, haben wir schließlich anstelle des Gefühls von Langeweile die Praktiken der Regungslosigkeit in den Blick genommen. ${ }^{17}$ Anfangs wurde das „Standby-Phänomen“ von uns Forschenden noch gar nicht wahrgenommen, die Beobachtungen mit der Kamera kreisten anhand unterschiedlicher Blickschneisen um die verkörperten Praktiken der Schüler/innen im Unterricht. Diese Konzentration auf „etwas“ hat den Rahmen dafür geschaffen, beim fokussierenden Schneiden auf einen „Standby-Modus“ zu stoßen.

Das publizierte Video spitzt die Beobachtung zu, indem ein auf den ersten Eindruck unpassender Text - ein befremdender Kommentar - an die Videosequenzen herangetragen wird: technische Definitionen zum Energiesparmodus von Computern. ${ }^{18}$ An diesem Beispiel entzünden sich regelmäßig Kontroversen: Darf man leibhaftige Schüler mit Computern vergleichen? Kann dies als wissenschaftliche Kommentierung gelten? Darf die Ethnographin Worte in den Mund nehmen, die dem Feld ,nicht auf der Zunge liegen“?

Der Standby-Kommentar leistet zweierlei: Er gibt einen direkten Hinweis auf den erarbeiteten ethnographischen Blick und sorgt zugleich dafür, dass Bild- und Tonspur sich nicht illustrativ verdoppeln, sondern ein vergleichendes interpretierendes Hinschauen provoziert wird. Mal öffnen Titel, Worte, Kommentare erst ein Verständnis der Bildfolgen, mal behindern sie dies. Entscheidend bleibt das Ziel, den Umgang mit audio-visuellem Material als Labor-

17 Es wäre dennoch reizvoll, gerade ein solches erkenntnisreiches Scheitern mit filmischen Mitteln zur Darstellung zu bringen.

18 Die Definitionen zum Begriff ,Standby“ stammen aus www.wikipedia.de. 
praxis der Herstellung von Sichtbarkeit und Sehen zu rahmen. ${ }^{19}$ Dies eröffnet Bezüge zur Frage nach dem Experiment in Geistes- und Naturwissenschaften und zur Rolle ästhetischer Erfahrung und Praxis beim Forschen. Das Aussetzen, Einsetzen und Auslösen von Worten kann genutzt werden, um ethnographische Erfahrungen machen und zeigen zu können.

Die Unterscheidung von Langer zwischen diskursiven und präsentativen Symbolmodi scheint mir an dieser Stelle hilfreich und in ihrer Konsequenz radikal. Das Ungenügen der Sprache liege darin, „dass wir unsere Ideen nacheinander aufreihen müssen, obgleich Gegenstände ineinander liegen; so wie Kleidungsstücke, die übereinander getragen werden, auf der Wäscheleine nebeneinander hängen. Diese Eigenschaft des verbalen Symbolismus heißt Diskursivität; ihretwegen können überhaupt nur solche Gedanken zur Sprache gebracht werden, die sich dieser besonderen Ordnung fügen; jede Idee, die sich zu dieser ,Projektion“ nicht eignet, ist unaussprechbar, mit Hilfe von Worten nicht mitteilbar“ (Langer 1984: 88).

Abbildung 9: Temporärer Körperraum

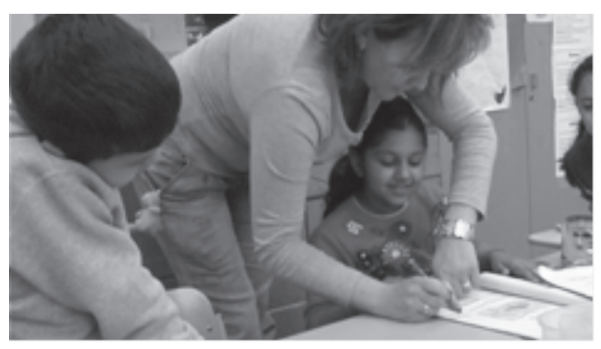

Abbildung 10: Temporärer Körperraum

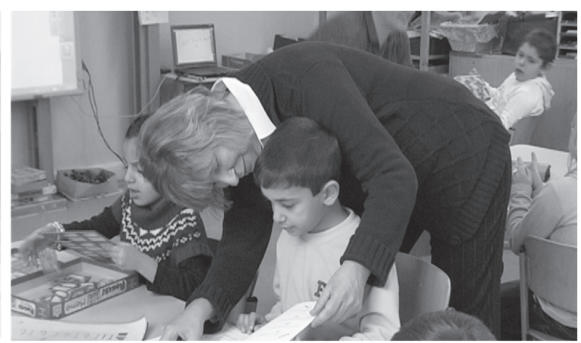

Quelle: DVD Mohn / Breidenstein (2013)

Mit einer kamera-ethnographischen „Werkstatt Wittgenstein wortlos“(WWw) schlage ich ein Projektformat vor, bei dem beobachtbare Praktiken im Rahmen von „Sprachspielen“ untersucht werden, zu denen jedoch die Worte (noch) fehlen. Begriffe im jeweiligen Sprachspiel zu beforschen steht im Zusammenhang einer Differenzierungsstrategie, bei der es darum geht, Phänomene in ihren verschiedenen Ausformungen in den Blick zu nehmen. Dabei erhalten nach Wittgenstein die aufgespürten Bedeutungsvarianten von Begriffen den Status von Zwischengliedern. Phänomen-Bereiche sollen zunächst für eine Vielfalt an Varianten geöffnet werden, anschließend lassen sich „Familienähnlichkeiten“ dieser Zwischenglieder entwerfen. Die Figur der Verknüpfung schafft ein offenes Netz möglicher Zusammenhänge, was Theoriearbeit an den Phänomenen bedeutet. „Es kommt darauf an, (interkulturelle) Phänomene in ihren unterschiedlichsten Aspekten als ,Zwischenglieder' so zu arrangieren, dass wir auch verdeckte oder ignorierte, unvermutete oder irritierende Zusammenhänge, Übergänge und Familienähnlichkeiten aufspüren können, ohne dabei einem wie auch immer definierten Wesensgehalt zuzustreben“, so Griesecke (2001: 4), die sich anhand der Philosophischen Untersuchungen (1949-1950) des späten Wittgensteins mit Theorien der Beschreibung befasst.

Ein anhand von Blickschneisen entstandenes visuelles Material eignet sich, den diskursiven um einen präsentativen Symbolmodus zu ergänzen, indem das Sichtbarmachen, Beobachten und Zeigen im gestaltenden Umgang mit dem Bildmaterial fortgesetzt wird: ein zerlegendes Differenzieren und ein montierendes Theoretisieren; das Aufspüren von Varianz und der Entwurf möglicher Zusammenhänge. Kontrastierende Collagen stellen den Aspekt-Charakter von

19 Hierin unterscheidet sich der kamera-ethnographische Ansatz von den Grundformen des Dokumentarischen, anhand derer T. Lipp (2012) diese fünf Prototypen des Dokumentarfilms unterscheidet: Plotbasierter Dokumentarfilm, Nonverbaler Dokumentarfilm, Documentary, Direct Cinema und Cinéma Vérité. 
Zwischengliedern heraus und können dabei gar etwas von dem zeigen, worüber wir nach Wittgenstein nur schweigen können.

„, Wittgenstein hat gesagt: ,Worüber wir nicht reden können, darüber müssen wir schweigen. 'Frederking (2003) zitiert einen anderen berühmten Wittgenstein-Satz: ,Die Grenzen meiner Sprache bedeuten die Grenzen meiner Welt" (Wittgenstein 1922: 36). Langer setzt dagegen: Die Grenzen der Sprache sind nicht die Grenzen der Erfahrung. Was nicht in das sprachliche Ausdrucksschema passt, ist nicht notwendigerweise, etwas Blindes, Unbegreifliches, Mystisches; es handelt sich einfach um Dinge, die durch ein anderes symbolisches Schema als die diskursive Sprache begriffen werden müssen' (Langer 1984: 95). Diese Erkenntnis ist die entscheidende Leistung Langers. “ (Wangerin 2004: 133)

Die Werkstatt Wittgenstein wortlos (WWw) setzt hier an und ich sehe darin ein vielversprechendes Experimentierfeld im Rahmen interdisziplinärer / kulturvergleichender Studien. Multimediale Installationen könnten denkbare Präsentationsformate solch komplexer Beobachtungsergebnisse sein. Eine WWw zu erproben und dabei Erkenntnispotentiale von Schnitt und Montage radikaler als im Rahmen bisheriger Auftragsstudien möglich zu nutzen, hierin sehe ich das aktuelle Anliegen kamera-ethnographischer Methodologie.

\section{Differenz der Situation}

Kamera-Ethnographie zu betreiben ist situiertes Forschen. Dabei reichen die Forschungssituationen über die eigentlichen Feldaufenthalte hinaus: Zeiten an Schreib- und Schneidetischen, in Seminar- und Vorführräumen gehören ebenso dazu. Die Situiertheit der beobachteten Praktiken und der Forschungspraxis überformen sich beim Feldaufenthalt und beides gerät dabei in die neue Konstellation einer Beobachtungssituation. Mit den Orten und Räumlichkeiten, in denen ethnographische Forschung stattfindet, hängen unterscheidbare Phasen im Wissensprozess zusammen, unterschiedliche situative Rahmungen mit verschiedenen Anfordernissen.

Im Ausbalancieren von Wahrnehmung und Vorstellung, Dokumentation und Visualisierung, Nicht-Wissen und Wissen gerät Kamera-Ethnographie beim Forschen in Paradoxien. Diese zu handhaben und zu nutzen, stellt eine methodologische Herausforderung dar. Oft wird versucht, sie anhand der Begriffe „Interpretation“ und „Dokumentation“ zu verhandeln. Konzepte zum „Dokumentieren“ loten die Spannungsfelder von Interpretation versus Dokumentation aus, umkreisen oder umgehen dabei die Frage nach dem Wissen und weichen in der Art, wie sie dies tun, auf brisante Weise voneinander ab. Dies habe ich anhand der vier Spielarten des Dokumentierens, die beim ethnographischen Forschen eine Rolle spielen, idealtypisch herausgearbeitet (Mohn 2002, überarbeitet 2011).

Von Spielarten zu sprechen ist Ergebnis einer Differenzierungsstrategie, bei der der Ausdruck Dokumentieren geöffnet werden soll für die Untersuchung empirisch auffindbarer Varianten. Dass man in lebensweltlichen Zusammenhängen immer auf solche Varianten stoßen wird, entspricht einer sprachphilosophischen Annahme. Seit Wittgenstein ist eine empirische Philosophie denkbar, die nicht mehr davon ausgeht, hinter die Phänomene blicken zu können, sondern die in der Beschreibung spezifischer (sprachlicher) Ausdrücke in ihrem Gebrauch die Möglichkeiten und Grenzen wissenschaftlichen Strebens sieht. Ähnlich verfährt Lynch (1993: 280ff), indem er vorschlägt, Begriffe aus der Erkenntnistheorie als „epistopics“ zu verstehen und ihren Gebrauch empirisch zu untersuchen. Dies sind Versuche der Spezifik wissenschaftlichen Wissens über eine Untersuchung kultureller Praxis näherzukommen. Meine Analyse der Konzepte des Dokumentierens legt in der Tat nahe, dass Dokumentieren dies oder jenes bedeutet, also so oder so „gespielt“ werden kann. Allerdings benenne ich die Differenzen im Hinblick auf unterschiedliche forschungspraktische Rahmungen. Dies schließt dann die Be- 
liebigkeit ihres Gebrauchs aus und zugleich eine Kenntnis des eigenen Forschungsprozesses ein.

Bei den vier Spielarten des Dokumentierens handelt es sich um Starkes Dokumentieren, die Dokumentarische Methode der Interpretation, Paradoxes Dokumentieren und Anti-Dokumentieren. ${ }^{20}$ Auf den ersten Blick schließen sich solche Konzepte aus. Doch anhand einer methodologischen Supervision lassen sich für jede der vier Spielarten unverzichtbare Effekte auf den Forschungsprozess herausarbeiten, denn im Versuch ihrer Befolgung mobilisieren sie Wissen oder Nichtwissen, Prozesswissen oder reflexives Wissen. Die vier Spielarten des Dokumentierens markieren sozusagen „Standpunkte im Sumpf“, die relativ schnell zu wechseln sind. Ziel ist es, Spannungsverhältnisse und Bewegungsmomente zwischen Nicht-Wissen und Wissen aufzuspüren, sich ihnen auszusetzen, sie aufrechtzuerhalten, einzurichten und zu gestalten. Dies setzt eine leicht distanzierte und latent reflexive Haltung zu den jeweils momentan gewählten methodologischen Strategien voraus.

Um die Zeitpunkte besser bestimmen zu können, an denen Strategien des Forschens gerade nutzen oder nicht mehr greifen, ist es hilfreich, Forschungsphasen entsprechend einzurichten, so dass sie Änderungen im Verhältnis von Wissen und Nicht-Wissen erfassen. Im Kontext kamera-ethnographischer Blickarbeit wird das verbreitete Zwei-Phasen-Modell „Datenerhebung versus Interpretation“ abgelöst durch sechs Forschungsphasen (vgl. Mohn 2010, 2011), die auf ethnographische Beobachtungsprozesse und das jeweils gewählte Medium prinzipiell übertragen werden können. Anhand methodologischer Register lässt sich eine Kopräsenz aller vier Spielarten des Dokumentierens vorstellen, transmethodologische Arrangements sozusagen, die sich von Phase zu Phase momentan passender Leitfiktionen (einer der Spielarten) unterordnen.

Abbildung 11: Heuristisches Modell der Kamera-Ethnographie zur Reflektion von Forschungsphasen, methodologischen Registern und Leitfiktionen:

\begin{tabular}{|l|l|l|}
\hline $\begin{array}{l}\text { Phase 1: } \\
\text { Blickschneisen }\end{array}$ & $\begin{array}{l}\text { Phase 2: } \\
\text { Versuchsanordnungen }\end{array}$ & $\begin{array}{l}\text { Phase 3: } \\
\text { Dichtes Fragen und Zeigen }\end{array}$ \\
\hline Nicht-Wissen & Nicht-Wissen & Nicht-Wissen \\
\hline Vor-Wissen & Vor-Wissen & Vor-Wissen \\
\hline Prozess-Wissen & Prozess-Wissen & Prozess-Wissen \\
\hline Reflexives Wissen & Reflexives Wissen & Reflexives Wissen \\
\hline
\end{tabular}

20 Starkes Dokumentieren fasst Strategien zusammen, die sich um eine Verzögerung des Sinnstiftens beim Forschen bemühen, um den Gegenstand in tieferer oder ungewohnter Bedeutung in den Blick zu bekommen. Hierein fallen sowohl die prominenten dokumentarfilmischen Konzepte der Offenheit und Leere, wie aber auch sozialwissenschaftliche Strategien blickfreier Datenproduktion durch die Unschuld der Technik. Die Dokumentarische Methode der Interpretation beschreibt die elementare Praxis blitzschnellen Immer-sofort-Wissens, über die alltägliche Verständigungsprozesse gerade deshalb gelingen, weil beim Deuten nicht gezögert wird. Wissenschaft, so sehr sie auch ihre Opposition zur Alltagspraxis herauskehrt, kann ohne dokumentarisches Interpretieren niemals gelingen. Paradoxes Dokumentieren kombiniert widersprüchliche Spielarten des Dokumentierens und befasst sich mit postmodernen Zwischenpositionen (weder noch) und oszillierenden Wechselspielen (sowohl als auch), die den Prozess in Bewegung halten bzw. nicht zum Abschluss kommen lassen. AntiDokumentieren schließlich stellt eine Gegenbewegung zum Verbergen der Autorschaft beim Starken Dokumentieren dar, will durch Reflexivität das Dokumentarische dekonstruieren, den wissenschaftlichen Autor wieder in den Blick rücken und in die Verantwortung nehmen, gerät dabei aber in einen Bumerang-Effekt, da sie dabei erneut dokumentiert, belegt und behauptet: So wird Wissenschaft gemacht! 


\begin{tabular}{|l|l|l|}
\hline $\begin{array}{l}\text { Phase 4: } \\
\text { Rezeption }\end{array}$ & $\begin{array}{l}\text { Phase 5: } \\
\text { Reflexion }\end{array}$ & $\begin{array}{l}\text { Phase 6: } \\
\text { Anwendung }\end{array}$ \\
\hline Nicht-Wissen & Nicht-Wissen & Nicht-Wissen \\
\hline Vor-Wissen & Vor-Wissen & Vor-Wissen \\
\hline Prozess-Wissen & Prozess-Wissen & Prozess-Wissen \\
\hline Reflexives Wissen & Reflexives Wissen & Reflexives Wissen \\
\hline
\end{tabular}

Quelle: eigene Zusammenstellung

Das heuristische Modell verfolgt den Gedanken einer Selbstanwendung der Ethnomethodologie, bei der Praktiken grundsätzlich im Kontext von Situationen und als Lösungen konkreter Handlungsprobleme betrachtet werden. Die über ein bildendes Forschen hinausweisende methodologische Innovation der Kamera-Ethnographie besteht darin, eine situierte Methodologie zu praktizieren: Ethnographisches Forschen findet an Orten und zu Zeiten statt. Von Situation zu Situation, von Phase zu Phase, von Forschungsort zu Forschungsort kann dabei recht Unterschiedliches bestens gelingen: konzentriertes Hinschauen; experimentelles Deuten; positioniertes Publizieren (in Worten oder Bildern); differenzierter Dialog; reflektierte Methodologie; forschende Praxisgestaltung.

Als heuristisches Modell ethnographischen Forschens (mit welchem Medium auch immer) hilft es, die tatsächlich praktizierten Vollzüge des Forschens jenseits reiner Methodenlehre zu begreifen. Praktiken in den unterschiedlichen Situationen des Forschens ethnographisch zu beobachten, um der „Fabrikation von Erkenntnis“ (Knorr Cetina 1984[1981]) in unseren eigenen Reihen auf die Schliche zu kommen, dürfte sich vor diesem Hintergrund lohnen.

„Schließlich liegt dem Ansatz der Grounded Theory die Annahme zugrunde, dass Forschung als Arbeit zu verstehen ist. Im Prinzip plädieren wir für eine in hohem Maße selbstreflexive Herangehensweise an die Forschungsarbeit, d.h. man muss sich überlegen, wie die Arbeit beschaffen ist und unter verschiedenen Bedingungen in den einzelnen Forschungsphasen durchgeführt werden kann." (Strauss 1991 / 1987: 34)

Die Überlegungen zur Kamera-Ethnographie als medienreflexive Variante der Grounded Theory Methodology zu betrachten und in diesem Rahmen zu betreiben, steht noch aus.

\section{Qualifizierte Differenz}

Die Entfaltung qualifizierter Differenz ist grundlegend für die Entwicklung einer zeigenden ethnographischen Forschung und ihr Potential, auch subtile Aspekte bis hin zu stummen Praktiken und Praktiken des Passiven thematisieren zu können. Kamera-Ethnographie betreibt eine praxeologische Ethnographie in Form von Entwurfsprozessen dichten Zeigens und Beschreibens. Es wird daran gearbeitet, das vor Augen Liegende durch Differenz überhaupt erst sehen zu können. Mithilfe einer interessiert hinschauenden Kameraführung werden Blicke und Bilder generiert, die Konzentration auf etwas erzeugen. Beim fokussierenden Videoschnitt werden die Verbildlichungsprozesse fortgesetzt. Den eigenen forschenden Blick als etwas Voraussetzungsvolles, Eingreifendes und Eingebundenes zu erfahren und in seiner Differenz und Gestaltungskraft zu nutzen, wäre ein Ausbildungsziel ethnographischer Professionskultur. Mir scheint es ein unterschätztes Qualitätsmerkmal zeigender Ethnographie zu sein, dass erst eine Positionierung der Blicke in ihrer Differenz zu den „Augen des Feldes“ ein Gegenüber schafft. Gibt sich der ethnographische Blick als differenter Blick zu erkennen und werden ethnographische Bildmaterialien als Manifestationen solch hausgemachter Blicke verstanden, dann eröffnet genau dies den Dialog über mögliche Sichtweisen und alternative Gestalten des Gezeigten. Bilder verkörpern Wissensweisen. Sie treffen einerseits Feststellungen, die anderer- 
seits anhand neuer Blicke und Fragen stets wieder aus der Fassung geraten können. Dann kann neu fokussiertes Bildmaterial entstehen, was darauf reagiert und in der Folge weitere Impulse gibt. Die Beobachter/innen bleiben im Spiel!

\section{Literatur}

Amann, K. / S. Hirschauer (1997): Die Befremdung der eigenen Kultur. Ein Programm, in: Dies. (Hrsg.), Die Befremdung der eigenen Kultur. Zur ethnographischen Herausforderung soziologischer Empirie, Frankfurt / Main, S. 7-25.

Astruc, A. (1964): Die Geburt einer neuen Avantgarde: Die Kamera als Federhalter, in: T. Kotulla (Hrsg.), Der Film. Manifeste, Gespräche, Dokumente (2). 1945 bis heute, München, S. 111-115.

Berg, E. / M. Fuchs (1993): Phänomenologie der Differenz. Reflexionsstufen ethnographischer Repräsentation, in: Dies (Hrsg.), Kultur, soziale Praxis, Text. Die Krise der ethnographischen Repräsentation, Frankfurt / Main, S. 11-108.

Blumenberg, H. (2001[1979]): Ausblick auf eine Theorie der Unbegrifflichkeit, in: Ders., Ästhetische und metaphorologische Schriften, Frankfurt / Main, S. 193-209.

Buchholz, M.B. (1999): Psychotherapie als Profession, Gießen.

Buchholz, M.B. (2005): Die Geburt der Sprache aus dem Geist der Musik. Evolutionstheoretische Überlegungen zum Verhältnis von Psychoanalyse und Musik, in: B. Oberhoff (Hrsg.), Die seelischen Wurzeln der Musik, Gießen, S. 87-122.

Clifford, J. / G. Marcus (Hrsg.) (1986): Writing Culture. The Poetics and Politics of Ethnography, Berkeley / CA.

Fleck, L. (1983[1936]): Das Problem einer Theorie des Erkennens, in: L. Schäfer / T. Schnelle (Hrsg.), Ludwik Fleck. Erfahrung und Tatsache, Frankfurt / Main, S. 84-127.

Fleck, L. (1983[1947]): Schauen, sehen, wissen, in: L. Schäfer / T. Schnelle (Hrsg.), Ludwik Fleck. Erfahrung und Tatsache, Frankfurt / Main, S. 147-174.

Geertz, C. (1987[1983]): Dichte Beschreibung. Beiträge zum Verstehen kultureller Systeme, Frankfurt / Main.

Geertz, C. (1990): Die Künstlichen Wilden. Anthropologen als Schriftsteller, München (zuerst 1988: Works and Lives. The Anthropologist as Author, Stanford / CA).

Griesecke, B. (2001): Japan dicht beschreiben. Produktive Fiktionalität in der ethnographischen Forschung, München.

Heath, C. (2012): Video Analysis and Organisational Practice, in: H. Knoblauch et al. (Hrsg.) 2012[2006], S. 35-49.

Hirschauer, S. (2001): Ethnographisches Schreiben und die Schweigsamkeit des Sozialen. Zu einer Methodologie der Beschreibung. Zeitschrift für Soziologie 30 / 6, S. 429-451.

Kalthoff, H. (2006): Beobachtung und Ethnographie, in: R. Ayaß / J. Bergmann (Hrsg.), Qualitative Methoden der Medienforschung, Reinbek / Hamburg, S. 146-182.

Knoblauch, H. (2012): Videography. Focused Ethnography and Video-Analysis, in: H. Knoblauch et al. (Hrsg.) 2012[2006], S. 69-83.

Knoblauch, H. / B. Schnettler / J. Raab (2012): Video-Analysis. Methodological Aspects of Interpretive Audiovisual Analysis in Social Research, in: H. Knoblauch et al. (Hrsg) 2012[2006], S. 9-26.

Knoblauch, H. / B. Schnettler / J. Raab / H.-G. Soeffner (Hrsg.) (2012[2006]): Video Analysis. Methodology and Methods, Frankfurt / Main.

Knorr Cetina, K. (1984[1981]): Die Fabrikation von Erkenntnis. Zur Anthropologie der Naturwissenschaft, Frankfurt / Main.

Knorr Cetina, K. (1988): Das naturwissenschaftliche Labor als ,Verdichtung' von Gesellschaft, in: Zeitschrift für Soziologie 17, S. 85-101. 
Krämer, S. (2001): Kann das ,geistige Auge' sehen? Visualisierung und die Konstitution empirischer Gegenstände, in: B. Heintz / H.D. Huber (Hrsg.), Mit dem Auge denken. Strategien der Sichtbarmachung in wissenschaftlichen und virtuellen Welten, Zürich, S. 347-364.

Krusche, J. / G. Vogt (2011): Strassenräume. Berlin, Shanghai, Tokyo, Zürich. Eine foto-ethnographische Untersuchung, Baden.

Langer, S.K. (1984[1942]): Philosophie auf neuem Wege. Das Symbol im Denken, im Ritus und in der Kunst, Frankfurt / Main (zuerst 1942: Philosophy in a New Key. A Study in the Symbolism of Reason, Rite and Art.)

Latour, B. (1979): An Anthropologist Visits the Laboratory, in: B. Latour / S. Woolgar, Laboratory Life. The Social Construction of Scientific Facts, London - Beverly Hills / CA, S. 43-90.

Latour, B. / S. Woolgar (1979): Laboratory Life. The Social Construction of Scientific Facts, Beverly Hills / CA.

Lynch, M. (1993): Scientific practice and ordinary action, Cambridge.

Mey, G. / K. Mruck (Hrsg.) (2011[2007]): Grounded Theory Reader, Wiesbaden.

Mohn, B.E. (1993): Ethnographische Visualisierung. Reflexionen anhand eines Videoprojektes in einem molekularbiologischen Labor, Magisterarbeit, Universität Frankfurt / Main.

Mohn, B.E. (2002): Filming Culture. Spielarten des Dokumentierens nach der Repräsentationskrise, Stuttgart.

Mohn, B.E. (2006): Permanent Work on Gazes. Video Ethnography as an Alternative Methodology, in: H. Knoblauch et al. (Hrsg.) 2012[2006], S. 173-181.

Mohn, B.E. (2007): Kamera-Ethnographie: Vom Blickentwurf zur Denkbewegung, in: G. Brandstetter / G. Klein (Hrsg.), Methoden der Tanzwissenschaft. Modellanalysen zu Pina Bauschs ,Sacre du Printemps', Bielefeld, S. 173-194.

Mohn, B.E. (2008 a): Die Kunst des dichten Zeigens: Aus der Praxis kamera-ethnographischer Blickentwürfe, in: B. Binder / D. Neuland-Kitzerow / K. Noack (Hrsg.), Kunst und Ethnographie, Zum Verhältnis von visueller Kultur und ethnographischem Arbeiten, Berliner Blätter 46, S. 61-72.

Mohn, B.E. (2008 b): Im Denkstilvergleich entstanden: Die Kamera-Ethnographie, in: E.O. Graf / B. Griesecke (Hrsg.), Ludwik Flecks vergleichende Erkenntnistheorie, Berlin, S. 211-234.

Mohn, B.E. (2010): Zwischen Blicken und Worten: kamera-ethnographische Studien, in: G.E. Schäfer / R. Staege (Hrsg.), Frühkindliche Lernprozesse verstehen: Ethnographische und phänomenologische Beiträge zur Bildungsforschung, Weinheim - München, S. 207-231.

Mohn, B.E. (2011): Methodologie des forschenden Blicks: Die vier Spielarten des Dokumentierens beim ethnographischen Forschen, in: P. Cloos / M. Schulz (Hrsg.), Kindliches Tun beobachten und dokumentieren, Weinheim - Basel, S. 79-98.

Mohn, B.E. (2012): Blick und Takt. Präsenz und Absenz von Takt in Schule, Kita und beim Forschen, in: G. Gödde / J. Zirfas (Hrsg.), Takt und Taktlosigkeit, Über Ordnungen und Unordnungen in Kunst, Kultur und Therapie, Bielefeld, S. 198-209.

Mohn, E. / K. Amann (1998): Forschung mit der Kamera, in: Anthropolitan 6, S. 4-20.

Mondada, L. (2012): Video Recording as the Reflexive Preservation and Configuration of Phenomenal Features for Analysis, in: H. Knoblauch et al. (Hrsg.) 2012[2006], S. 51-67.

Moritz, C. (2009): Die Feldpartitur. Mikroprozessuale Transkription von Videodaten über einer Zeitachse, abrufbar unter: www.qualitative-forschung.de/methodentreffen/archiv/poster/poster 2009/moritz.pdf, letztes Abrufdatum: 20.3.2013.

Strauss, A. (1991[1987]): Grundlagen qualitativer Sozialforschung. Datenanalyse und Theoriebildung in der empirischen soziologischen Forschung, München.

Streeck, J. (2007): Hände, Handeln, Händel, in: B.E. Mohn / J. Wiesemann, Begleitpublikation zur DVD Handwerk des Lernens. Kamera-ethnographische Studien zur verborgenen Kreativität im Unterricht, Göttingen. 
Wangerin, W. (2004): Die Grenzen der Sprache sind enger als die Grenzen der Erfahrung. Was Susanne K. Langers und Alfred Lorenzers Symboltheorie für eine kreative Mediendidaktik bedeuten kann, in: V. Frederking (Hrsg.), Lesen und Symbolverstehen, Jahrbuch Medien im Deutschunterricht 2003, S. 128-139.

Weingarten, E. / F. Sack (1979[1976]): Ethnomethodologie. Die methodische Konstruktion der Realität, in: Dies. / J. Schenkein (Hrsg.), Ethnomethodologie. Beiträge zu einer Soziologie des Alltagshandelns, Frankfurt / Main, S. 7-26.

Wittgenstein, L. (1989-1993): Werkausgabe in 8 Bänden, Frankfurt / Main.

Wittgenstein, L. (1996[1922]): Tractatus Logico-philosophicus, in: J. Kelly (Hrsg.), Ludwig Wittgenstein. Ein Reader, Stuttgart.

\section{Visuelle Studien (DVDs)}

Mohn, B.E. (2011): DVD Rezeption machen. Beobachtungen zur Rezeptionsforschung im Theater für Kinder, Göttingen.

Mohn, E. / K. Amann (2006): DVD Lernkörper. Kamera-Ethnographische Studien zum Schülerjob, Göttingen.

Mohn, B.E. / G. Breidenstein (2013): DVD Arbeitswelten in der Grundschule. Praktiken der Individualisierung von Unterricht, Göttingen.

Mohn, B.E. / S. Hebenstreit-Müller (2007): DVD Kindern auf der Spur. Kita-Pädagogik als Blickschule. Kamera-Ethnographische Studien 1 des Pestalozzi-Fröbel-Hauses Berlin, Göttingen.

Mohn, B.E. / G. Wartemann (2009): DVD WECHSELSPIELE im Experimentierfeld Kindertheater. Reihe Kamera-Ethnographische Studien, Göttingen.

Weitere Video-Studien der Autorin: siehe www.kamera-ethnographie.de

Dr. Bina Elisabeth Mohn

Zinzendorfstraße 4

10555 Berlin

e.mohn@onlinehome.de

www.kamera-ethnographie.de 